http://jmscr.igmpublication.org/home/ ISSN (e)-2347-176x ISSN (p) 2455-0450 crossref DOI: https://dx.doi.org/10.18535/jmscr/v8i10.34

\title{
Artesunate: an Artemisinin Derivative having Antiviral Properties with Multiple Pleotropic Effects is a Perfect Potential Agent for the Treatment of Symptomatic COVID-19 Infection and Related Hyper inflammation States
}

\author{
Authors \\ Dr (Prof.) Butungeshwar Pradhan*, Dr (Asst. Prof.) Bikash Chandra Nanda, \\ Dr Gourav Pradhan \\ VSSIMSAR, Burla, Sambalpur, Odisha, India Pin-768017 \\ *Corresponding Author \\ Dr (Prof.) Butungeshwar Pradhan
}

\begin{abstract}
Artemisinin are not limited to the treatment of malaria, and wealth of studies demonstrating the activity of artemisinin and its derivatives against cancer cells, anti-inflammatory effects against SLE, RA, allergic asthma and as antiviral agent in various viral diseases including single stranded RNA virus Hepatitis $C$. Artesunate has concentration dependent killing effects on malaria and other viral infections with autoinduction of its own metabolism. Thus, initial high IV doses, short course of Artesunate therapy may be very useful as an antiviral agent in the treatment of COVID-19 infection. Artemisinin class of compounds have several multiple beneficial effects that are important for different diseases, might be more versatile and more successful. Artesunate a multifunctional molecule with favourable PK/PD characteristics, is a perfect potential option for urgent treatment of symptomatic COVID-19 infection. Due to its anti-inflammatory, immunomodulatory, antioxidant, anticytokine, multiorgan protective properties and antiviral activity against wide varieties of viruses including SARS-CoV-2 in-vitro. It has also synergistic effects with other antiviral drugs; decreases viral replication and decreases TNF- $\alpha$ and IL-6 levels, which are key in COVID-19 induced cytokine storm and ARDS.

Keywords: Artesunate, Antiviral, COVID-19, Anti-inflammatory, Anticytokine, immunomodulatory, Antioxidant, IL-6, TNF- $\alpha$.
\end{abstract}

\section{Introduction}

Clinical features of SARS-CoV-2 such as fever, sore throat, dry cough; myalgia and dyspnoea are common in early COVID-19 infection and progress rapidly to pneumonia and ARDS. SARS-CoV-2 selectively induces high levels of IL-6 and results in exhaustion of lymphocytes. COVID-19 cytokine storm associated with increased CRP, neutrophils, procalcitonin levels and other inflammatory indexes are increased in severe COVID-19 patients, such as chemokines CXCL-10, CCL-2. There is T cell over activation i.e. Th17 cells and increased high toxicity of CD8+ $\mathrm{T}$ cell. In addition there are increased levels of cytokines and chemokines and decreased levels of anti-inflammatory cytokine such as IL-10. In fatal SARS-CoV-2 there occur increased levels of IL-6, IL-1 $\beta$, IFN and CXCL-10 secreted by dendritic cells (DCs) and macrophages. Innate immunity play important role by inducing the infiltration and recruitment of inflammatory Th17 
cells. In peripheral blood CD4+, CD8+ cells are decreased and increased concentration of proinflammatory c-c Chemokines receptor (CCR4+, CCR6+) and Th17 cells are found in COVID-19 patients. $^{1}$

\section{Anti-inflammatory,}

Antiviral, immunomodulatory, anti-cytokine, and antioxidant effect of artemisinin derivatives:

Artemisinin (ARTs) family of drug regulates immune cells and their molecular mechanisms. NLRP3 is a sensor component expressed mainly in macrophages, which undergoes transcription by NF$\mathrm{k} \beta$. NLRP3 is responsible for maturation and secretion of IL-1 $\beta$, IL-18. NF-k $\beta$, also increases the levels of IL-10 in macrophages infected by pf malaria and ARTs can decrease IL-10 production in animal and human. ART family of drugs regulates cells from the innate and adaptive immune system and leads to anti-inflammatory, immunomodulatory activity. Artesunate (AS) and other ARTs can decrease pathologic changes and neutrophils infiltration to the lung of ALI patients and decreases sepsis-induced mortality by inhibiting NF-k $\beta$ signaling and enhance heme oxygenase-1 expression. AS can lower the concentration of TNF$\alpha$ and IL-6 in serum and bronchoalveolar lavage fluid (BALF). Dihydroartemisinine (DHA) a metabolite of AS attenuates LPS-induced ALI through suppression of NF-k $\beta$ signaling in a nuclear factor erythroid-2-related factor-2(Nrf2) dependent fashion leading to decrease in expression of proinflammatory cytokine IL-1 $\beta$, TNF- $\alpha$ and IL-6.In burn-based sepsis of BALB/L model, concentration of adhesion molecules and neutrophils infiltration in the lung, heart increased and there is increase mortality which is reverse by ARTs. ARTs down regulate protein levels of NOD, LRR and pyrin domain containing protein (NOD) 2 and macrophage. Toll-like receptor-2(TLR2) mainly located outside the cell membrane of macrophages, DCs, and granulocytes and recognized by bacteria and viruses. TLR2 induces NF-k $\beta$ activation through recruitment of TIR domain containing adaptor protein (TIRAP) and myeloid differentiation primary response (MYD) 88 in macrophages and DCs. In inflammatory monocytes, TLR2 expressed within endosomes and induces the release of type 1 IFNs via interferon regulatory factor-3(IRF-3) and IRF7 in response to viruses. Artesunate increases survival of mice by reducing TNF- $\alpha$ in methicillin resistant staphylococcus aureus bacterial infection and decrease TLR2 mRNA and NOD2 mRNA and decrease NF-k $\beta$. AS decreases TNF- $\alpha$ and IL- 6 from macrophages by inhibiting TLR-4 mediated auotophagic activation.TLR4 also located in endolysosomal compartment can recognize gram-negative bacteria and viruses, share the same pathway as the activation of NF-k $\beta$ and induces release of type 1 IFNs via the TNF receptor associated factor [TRAF30-TANK binding kinase-1(TBKI)-1-IRF3] Axis. AS attenuates cytokine release by the TRAF6beclin1-class II phosphatidylinositol-3-kinase (P13KC3) pathway. In a severe acute pancreatitis model; AS attenuates release of IL- $1 \beta$ and IL- 6 via the TRL4-NF-K $\beta$ axis. DHA inhibits activation of TRL4 and IRF3 in the spleen cells of SLE protein $\mathrm{MRL} / 1 \mathrm{pr}$ mice, which leads to decrease in levels of IFN- $\alpha$ and IFN- $\beta$. AS also lower IL-1 $\beta$, IL-2, IL-6, IL-8, IFN-y, CCL2, CCL3 and CCL4 in mice infected with herpes simplex virus. AS down regulate $\mathrm{Th}_{1}$ response and decreases IFN-y, TNF- $\alpha$, IL-12， IL-18， CCL2， CXCL9， CXCL10 in experimental cerebral malaria. In clinical study of malaria AS decreases IL-6 and soluble IL-6 receptor in plasma and normalized within 24 hours. ${ }^{1}$

Artesunate is effective against Hepatitis B virus and has synergistic effect with Lamivudine. Artesunate has highest antiviral effects against human cytomegalovirus (HCMV) higher than gangcyclovir and synergistic with Lamivudine, cedofovir and gangcyclovir. Artesunate has the highest antiviral activity against HCMV among the derivatives of artemisinin. Artesunate + valcyclovir are effective against Herpes simplex encephalitis. Artesunate has anti-inflammatory effects and lower IL-1B,IL-2,IL$6, \mathrm{IFN}-\gamma$ and $\mathrm{CC} 2$ treated with valcyclovir versus valcyclovir alone.AS also effective against Epstien Barr virus (EBV), Human herpes simplex-6 (HHP6), human BK polyomavirus (BKPyV) which 
causes nephropathy, and JC polyoma virus (JCPyV) which causes progressive multifocal leukoencephalopathy (PML). Artemether is most effective against HCMV and Ebola virus (EBOV). DHA effective against bovine diarrhea virus (BVDV). ${ }^{2}$

Artesunate may inhibit NF-k $\beta$ downregulation and viral protein synthesis, disrupting the early phase of viral replication. Artesunate has been shown to inhibit the reproduction of hepatitis $B$ virus in vitro and inhibits the replication of hepatitis $\mathrm{C}$ replicon which is just like SARS-CoV-2 a single-stranded RNA virus. Artesunate thought to inhibit IL-1 $\beta$, IL6 and IL-8 production through inhibition of the NF$\mathrm{kB}$ signal pathway. Artemisinin and its derivatives have anti-inflammatory and immune regulatory effects and to be effective in systemic lupus erythematosus, rheumatoid arthritis and allergic contact dermatitis and bronchial asthma. Artemisinin and its derivatives suppress the production of IL-2; inhibit nitric oxide synthease and NF- $\mathrm{BB}$ activation, thereby providing the treatment of rheumatoid arthritis. Artesunate can regulate the effects of regulator $\mathrm{T}$ cells via NF$\kappa \mathrm{B} / \mathrm{p} 65$ and Smad2/3-dependent TGF- $\beta$ (Transforming growth factor beta) signaling. Artesunate showed anti-inflammatory activity by decreasing in TNF- $\alpha$ and IL-6 levels. Artesunate also significantly reduce the expression of MCP-1 and TNF- $\alpha$ in serum. ${ }^{3}$

Artesunate inhibit TNF- $\alpha$ induced production of IL$1 \beta$, IL-6, and IL-8 in RA fibroblast-like synoviocyte in a dose dependent manner. Artesunate also inhibit TNF- $\alpha$ induced NF-k $\beta$ translocation of DNA binding activity and gene transcription activity as well as phosphorylation and degradation activity of $\mathrm{Ik} \beta$. Artesunate inhibit production of IL-1 $\beta$,IL- 6 and IL-8 through inhibition of NF-k $\beta$ signaling pathway, prevent Akt phosphorylation suggesting that inhibition of Akt activation might inhibit IL-1 $\beta$,IL-6 and IL- 8 production induced by TNF- $\alpha$. Akt a serine threonine protein kinase a downstream effector of $\mathrm{p} 13 \mathrm{k}$. Activation of $\mathrm{p} 13 \mathrm{k}$ increases the intracellular amount of phosphatdylinositol-3, 4, 5-triphosphate, which positively induces Akt phosphorlation. The p13k / Akt pathway, can be activated by several cytokines such as TNF- $\alpha$, TGF- $\beta$; and IL-17 in RA synoviocytes. The $\mathrm{p} 13 \mathrm{k} / \mathrm{Akt}$ pathway in addition to anti-apoptottic mediates production of same proinflammatory cytokines production in synoviocytes. Artesunate inhibit TNF- $\alpha$-induced Akt phosphorylation. AS also modulate TNF- $\alpha$ induced proinflammatory cytokine synthesis via at least in part the p13k/Akt pathway. Thus, artesunate has anti-inflammatory effects and useful in RA. ${ }^{4}$ In clinical study of severe malaria, AS decreases IL-6 and soluble IL-6 receptor in plasma and normalized within 24 hours. ${ }^{5}$

Artesunate has antiviral effect by inhibiting HCMV through a reduction in the DNA binding activity of NF-k $\beta$ and Sp1 and subsequently downstream activity of Akt1 and P70S6K. Artesunate effects in vitro are inhibition of cell proliferation, induction of cell cycle arrest, and apoptosis, inhibition of inflammation and oxidation stress, inhibition of angiogenesis, invasion and metastasis and chemosensitization of cancer cells to therapeutic agents. Artesunate is not toxic to healthy and nondisease cells. Thus, it has effect only on cancer cell. Anti-proliferative effects of AS in non-cancer cells are by reduction in $\mathrm{p}$-Akt and $\mathrm{p}$ - $\mathrm{p}-70 \mathrm{~s} 6 \mathrm{k}$ protein expression. Artesunate could mitigate P13K/Akt and p44/42 mitogen activated protein kinase (MAPK) signaling pathways that are activated in COPD. Pretreatment with artesunate reduces LPS induced IL-6 generation with no effect on cell viability. Artesunate mitigated hypoxia/ reoxygenation-induced increase in ROS levels in alveolar macrophages and oxidative stress, together with reduction in NLR family pyrin domain containing (NLRP3) and apoptosis associated speck-like protein contains CARD (ASC) protein abundance caspase- 1 activity and production of IL$1 \beta$, IL-18 mRNA and protein levels. Studies in bleomycin-induced lung injury, fibrosis, ALI, asthma and COPD, DHA increases lung tissue mRNA and protein levels of nuclear factor erythroid-2 related factor 2 (Nrf2) and oxygenase1(HO-1) due to increase SOD and GSH and decrease malondialdehyde (MDA). Artesunate 
increase activation of Nrf2 leading to mRNA expression. Artesunate also increase Nrf2 in LPSinduced increase in Nrf2 and HO-1 expression in ALI.AS decreases the expression of oxidative damage marker 8-isoprostane, 8-hydroxy-2deoxyguanosene (8-HdG) and 3-nitrotyrosine (3NT) and gene expression of regulatory subunit of NADPH oxidase (NOX) 2, NOX4, P22phox and p67phox. Expression of SOD, inducible Nitric oxide synthease (iNOS) and NOX1-4 were decreased, while catalase levels increases. In smoking induced lung oxidation damage AS stimulate Nrf2 expression, while reducing SOD activity, 3-NT, and MDA. In COPD artesunate dose dependently ablated expression of 8-isoprostane, $80 \mathrm{HdG}$ and 2NT in BALF increases catalase activity and decrease NOX2 protein expression in mice lung. AS also attenuates lung injury in paraquat-intoxicated rats via reduction in TGF $\beta 1$, IL-10 and TNF- $\alpha{ }^{6}$

Artesunate dose-dependently inhibits ovalbumin (OVA)-induced increased total eosinophil counts, IL-4, IL-5, IL-13 and eotaxin levels in BALF in female BALB/c mice with ovalbumin developed airway inflammation. AS attenuates lung tissue eosinophilia and airway mucus production, mRNA, E-selectin expression, IL-17, IL-33 and Muc5ac in lung tissues and airway hyperresponsiveness to methacholine. In normal human bronchial epithelial cells, artesunate blocks epidermal growth factorinduced phosphorylation of Akt and its downstream substrates tuberin p70S6 kinase and 4E-binding protein-1 and transactivation of NF-kB. Similarly, artesunate blocks the phosphorylation of Akt and its downstream substrates in lung tissues from Ovalbumin challenged mice. Artesunate ameliorates experimental allergic airway inflammation probably via negative regulation of PI3K/Akt pathway and the downstream NF-kB activity. ${ }^{7}$

Artesunate inhibit TNF- $\alpha$ and IL- 6 release from RAW264.7 cells mouse bone marrow-derived monocytes (BMDMs) and peritoneal macrophages (PMs) in lipopolysaccharide (LPS) induced sepsis. Artesunate inhibit LPS-induced cytokine release and autophagic activation via TLR4-TRAF6 signaling.AS could reduce the levels of TRAF6,
Beclin1, and PI3KC3. This interrupted the TRAF6Beclin1 interaction and subsequent formation of Beclin1-PI3KC3 core complex of the PI3K-III complex. This effect was tightly related to blockade of the TRAF6-Beclin1-PI3KC3 pathway via decreasing K63-linked ubiquitination of Beclin1 and then interrupting the formation of Beclin1PI3KC3 core complex of the PI3K-III complex. These findings reveal the anti-inflammatory effect of $\mathrm{AS}^{8}{ }^{8}$

Artesunate decreases acute inflammatory response through activation of Nrf2 pathway in bleomycin induced ALI, decrease the number of inflammatory cells (PMNs), macrophages and lymphocytes together with IL-4, IL-6, Tumor growth factor$\beta$ (TGF- $\beta$ ) and monocyte chemoattractant protein1(MCP-1) mRNA expression. Artesunate in $30 \mathrm{mg} / \mathrm{kg}$.b.w dose decreases total number of eosinophils and neutrophils inflammatory cell count in OVA -induced model of allergic asthma and decreases IL-8 and total inflammatory cells in cigarette smoke induce lung oxidative stress in mice. Pretreatment with Artesunate reduce LPSinduced ALI and in renal ischemia/reperfusion (I/R)-induced total inflammatory cell count, macrophages, neutrophils and IL-1 $\beta$ levels by NLRP3 activation. In addition it decreases IL-6, TNF- $\alpha$, NF-k $\beta$ and TLR4 levels in LPS-induced ALI and IL-18 levels in I/R-induced ALI. The activation of NLRP3 inflammasome was dependent on pulmonary ROS generation accompanied by higher ASC and caspase-1 levels. AS suppress I/Rinduced inflammation including production of serum and pulmonary NO, MDA, macrophage inflammatory protein- 2(MIP-2) and prostaglandin E2 (PGE2) and attenuated NF-k $\beta$ translocation. AS also protect sepsis induced lung injury by reducing IL-6, TNF- $\alpha$ in both serum and BALF. In the lung suppresses cyclooxygenase-2(COX-2), iNOS and $\mathrm{NF}-\mathrm{k} \beta$ levels and activated Nrf2 through increase in HO-1 expression and enzyme activity.AS decreases fibrosis, angiogenesis and metastasis, decreases mucous production and tumor proliferation and have airway remodeling effects.AS decreases IL-4, IL-6,IL-8,IL-10,IL-18,NLRP3,ASC,IL1 $\beta$, 
Neutrophils, macrophage, lymphocyte infiltration, TNF- $\alpha$, TGF $\beta$, MCP-1,NF-k $\beta, C O X-2, \quad$ iNOS,ROS, NLRP3, ASC, increase Nrf2, and HO-1.Thus, AS may be effective against COVID-19 induced ALI/ARDS.

The concentration of DHA for airway remodeling effect is about $5.7 \mu \mathrm{M}$. Anti-inflammatory, antioxidant effect of AS is $5.7 \mu \mathrm{M}$ and it is about $30 \mu \mathrm{M}$ for DHA. ARTs in larger doses and higher concentrations are required for cell proliferation, apoptosis and moderate doses for antiinflammatory, anti-angiogenesis, invasion and metastasis effects, while lower doses are required for airways remodeling and lung function studies. The effective plasma concentration of AS for modulation of lung function is about $2.5 \mu \mathrm{M} .^{9}$

\section{Artesunate in clinical viral infectious diseases}

The bioactivity of Artesunate has broader effects against viruses and includes inhibition of certain viruses, such as HCMV, EBOV, EBV; other members of the Herpesviridae family, hepatitis B virus ( $\mathrm{HBV}$ ), hepatitis $\mathrm{C}$ virus ( $\mathrm{HCV})$, and bovine viral diarrhea virus. The herpesviruses that have been analyzed thus far have all demonstrated similar sensitivity to artesunate (IC50, <10 $\mu \mathrm{M}$ ). Artesunate has more antiherpes viral potency than does its parental drug artemisinin.

1. Artesunate in human cytomegalovirus (HCMV) replication is connected with NF-KB and Sp1 activation pathway and cellular signaling kinase phosphoinositol 3 kinase. Phosphoinositol-3 kinase is required for the activation of NF-k $\beta$ and Sp1. The phosphorylation of downstream effectors of phosphoinositol-3- kinase such as protein kinase Akt and P70s6k is also inhibited by artesunate. The HCMV immediate-early promoter enhancer contains binding sites for both $\mathrm{Sp} 1$ and NF-kB , therefore, is responsive to both factors. Reduction of IE2p86 expression critically limits viral replication, because IE2p86 is essential for the initiation of subsequent regulatory steps. On the other hand, NF$\mathrm{kB}$ is a major factor in cellular defense pathways and may also have a negative impact on viral productivity and the course of infection. Thus, the activation pathways that involve $\mathrm{Sp} 1$ and NF-kB are important factors in the initial onset of the viral replication cycle, as well as in later steps in viruscell interaction, and therefore, crucial for the antiviral action of artesunate. Increase intracellular iron concentration enhance the artesunate's antiCMV activity (ferrous form), and/or soluble Transferrin resulted in enhanced suppression of viral replication and synergistic effects with gangcyclovir and cidofovir and foscarnet. A possible mechanism was suggested by the finding that artesunate inhibited central regulatory processes of HCMV infected cells (such as activation pathways dependent on NF-kB or Sp1), thus interfering with critical host-cell-type and metabolism requirements for HCMV replication. For both NF-kB and Sp1, a reduction in HCMV induced protein synthesis and a reduction in the DNA binding activity of NF-kB and Sp1 were observed with artesunate treatment.

2. The concentration at which Artesunate was active against $\mathrm{HBV}$ is highly species specific viruses, that have a virus-encoded DNA polymerase with reverse transcriptase activity and artesunate at the plasma concentration of $10 \mu \mathrm{M}$ was reported for its activity against HCMV and have synergistic effect with lamivudine. Interestingly, these levels are close to the drug concentrations achieved in the plasma of patients in whom this drug is used for anti-malarial treatment $(\sim 7 \mu \mathrm{M})$ and thus, concentration of artesunate required for antiviral effect is $(>10 \mu \mathrm{M})$.

3. HCV belongs to family of Hepacivirus is a single stranded RNA virus, similar to SARS-CoV2.Artesunate inhibit $\mathrm{HCV}$ replication by dosedependently in $2 \mathrm{HCV}$ subgenomic replicon constructs at concentrations that did not affect Huh 5-2 host cells. Hemin an iron donor inhibits HCV replicon replication by inhibiting viral polymerase. ${ }^{\mathbf{1 0}}$

\section{Renoprotective effect of Artesunate}

DHA also lessened glomerular injury and relive increase in urinary albumin: creatinine ratio and decreases serum levels of creatinine. Artesunate $(28.8 \mathrm{mg} / \mathrm{kg})$ reduced proteinuria and preserved renal function in nephritis mice model. Artesunate attenuated TNF- $\alpha$ and IL-6 levels, suppressed $\alpha$-SMA, TLR4, MyD88, NF- $\kappa$ B p65 and TGF- $\beta 1$ 
protein expression, and decreased caspase-3 activity in nephritis mice. Artesunate may prevent nephritis and inhibit inflammation via the TLR4/NF- $\kappa \mathrm{B}$ signaling pathway in mice. Both natural and acquired immune responses against pathogens are mediated by Toll-like receptors (TLRs). After TLRs recognize pathogen-associated molecular patterns, signals are transmitted into the cellsvia the Toll-interleukin (IL) -1 receptor homology region (TIR), to activate NF- $\mathrm{BB}$ transcription factors, causing the release of various immune-associated cytokines, including IL-1, IL-6, IL-8 and TNF- $\alpha$, thus causing an inflammatory response. Artesunate attenuated TNF- $\alpha$ and IL- 6 levels, suppressed $\alpha$-SMA, TLR4, MyD88, NF- $\kappa$ B p65 and TGF- $\beta 1$ protein expression, and decreased caspase- 3 activity in nephritis mice. ${ }^{11}$

\section{Artesunate effectiveness in clinical trial of COVID-19 patients:}

In a prospective study of 43 cases of confirmed COVID-19 patients enrolled and divided into routine treatment group $(\mathrm{n}=25)$ and Artesunate treatment group $(\mathrm{n}=18)$ by odd-even rule. The routine treatment group received lopinavir/ritonavir $500 \mathrm{mg}+\alpha$-aerosolized interferon $500 \times 104 \mathrm{U}$ twice daily; the Artesunate treatment group was given Artesunate $60 \mathrm{mg}$ twice daily, besides the routine treatment for 10 days. In Artesunate treatment group, the time for significant improvement of symptoms was (days: $3.33 \pm 1.91$ vs. $4.84 \pm 2.19$ ), negative conversion time of COVID-19 nucleic acid was (days: $4.72 \pm 2.16$ vs. $6.68 \pm 3.76$ ), lung lesion absorption starting time (days: $5.39 \pm 2.36$ vs. $7.48 \pm 3.78$ ), lung lesion absorption greater than $70 \%$ time (days: $14.11 \pm 4.16$ vs. $17.04 \pm 4.42$ ) and the length of hospital stay (days: 16.56 \pm 3.71 vs. $18.04 \pm 3.97$ ) were significantly shorter than those in routine treatment group, with significant differences (all $\mathrm{P}<0.05$ ). Artesunate shorten the treatment time of COVID-19, improved prognosis and eliminated pathogens, with fewer adverse reactions and a good application prospect. $^{12}$ In this trial low dose artesunate had been used and whatever benefits incurred may be only due to artesunate's antiinflammatory, anticytokine and antioxidant effects.
Similarly in the NCT04387240 trial, in which oral artesunate $100 \mathrm{mg}$ daily will be used for 5 days in mild to moderate adult cases will have less antiviral effects.

Drug exposure level (auc) and time effect on efficacy of artesunate in malaria:

AS and DHA has concentration dependent killing effect of malaria parasites. DHA is a metabolite of artesunate is highly effective against almost all stages of parasites achieving $100 \%$ growth inhibition within 2-4 hrs of exposure. ${ }^{13^{\prime} 14}$ In-vitro dose range of artesunate of $10 \square-10 \square$ M (30$3000 \mathrm{ng} / \mathrm{ml}$ ) is extremely effective against rings to early schizonts and $100 \%$ inhibition of parasites growth within 4-6 hrs. At drug concentration of $10 \sqsupset-10^{-5} \mathrm{M}(280-2800 \mathrm{ng} / \mathrm{ml})$ requires $3 \mathrm{hrs}$ of exposure time to show parasiticidal effect and at $10^{-}$ $7 \mathrm{M}(28 \mathrm{ng} / \mathrm{ml})$ requires $24 \mathrm{hrs}$ of exposure time for cidal effect. ${ }^{15}$ At $10^{-}{ }^{8}-10^{-} \square \mathrm{M} \quad(3-30 \mathrm{ng} / \mathrm{ml}$ ) requires 24-96 hrs of constant exposure to kill all parasites (Ic 99). At $10^{-8} \mathrm{M}(2.8 \mathrm{ng} / \mathrm{ml})$ had no appreciable effect on parasites. Thus, low dose artesunate will increase the treatment period and higher dose will shorten the treatment period. Therefore, low dose artesunate regimens cannot be selected for treatment of COVID-19 and lower dose longer exposure time is the principal factor leading to fatal neurotoxicity with Arteether and Artemether. ${ }^{16}$ Thus, artesunate plasma concentrations should be much more in the range of $10 \sqsupset-10^{-5} \mathrm{M}$ (300-3000ng/ml)or even higher for first exposure to virus infected cells. ${ }^{15}$

\section{Pharmacokinetic (PK) and pharmacodynamic} (PD) of artesunate

The PK/PD of Artesunate derived from treatment of malaria can be applied to treatment of COVID-19 infection. Artesunate at ( $2 \mathrm{mg} / \mathrm{kg} \mathrm{b.w.)} \mathrm{IV} \mathrm{dose:}$ Its Peak plasma concentrations of $2640 \mathrm{ng} / \mathrm{ml}$ have been reported and $2020 \mathrm{ng} / \mathrm{ml}$ for its active metabolite DHA. After $2.4 \mathrm{mg} / \mathbf{k g}$ IV dose of AS: this current $120 \mathrm{mg}$ of initial IV dose of AS produce a Cmax of $11343 \mathrm{ng} / \mathrm{ml}$ with $\mathrm{t}^{1 / 2}$ of $0.05 \mathrm{hrs}$ and Cmax of DHA 2646ng/ml with $\mathrm{t}^{1 / 2}$ of $0.67 \mathrm{hrs}$ (total $13987 \mathrm{ng} / \mathrm{ml})$. Intravenous AS is very fast and efficient on killing malaria parasites at a mean time 
of $3.1 \mathrm{hrs}$ for clearance of half of parasites (Pc 50) is the lowest Pc 50 than other artemisinin. ${ }^{17}$ After 2.4mg/kg dose of IM AS: The Cmax of AS was $532-3211 \mathrm{ng} / \mathrm{ml}$ (median $2193 \mathrm{ng} / \mathrm{ml}$ ) with $\mathrm{t} 1 / 2$ of 30 min, and rapidly hydrolyzed to DHA Cmax of 488$2011 \mathrm{ng} / \mathrm{ml}$ with $\mathrm{t}^{1 / 2}$ of $52 \mathrm{~min}$ The Cmax of artesunate was $42 \mu \mathrm{M}$ following a single intravenous injection dose of $120 \mathrm{mg}$, which is greater than the EC50 of $13.31 \pm 1.24 \mu \mathrm{M}$ of DHA against SARS$\mathrm{CoV}-2$,indicating that artesunate is a potential countermeasure against COVID-19. ${ }^{18}$ After $100 \mathrm{mg}$ of oral AS: The Cmax of AS was $198 \mathrm{ng} / \mathrm{ml}$ and DHA $1052 \mathrm{ng} / \mathrm{ml}$ with $\mathrm{t} 1 / 2$ of $0.75 \mathrm{hrs}$ (39-95 min) versus $3.2 \mathrm{mg} / \mathrm{kg}$ of IM Artemether: produce Cmax of AS $171 \mathrm{ng} / \mathrm{ml}$ with $\mathrm{t}^{1} / 2$ of $10 \mathrm{hrs}$ and little conversion to DHA. Thus, IV Artesunate is the choice of therapy. ${ }^{19^{\prime} 20}$ The IV AS can provide sufficiently high peak concentrations, provide fastest efficacy in killing malaria parasites, and IV injectable AS is superior to other artemisinin PK/PD in various regimens. The current dose of AS $120 \mathrm{mg}(2.4 \mathrm{mg} / \mathrm{kg})$ results in the Cmax ranging from $605-18909 \mathrm{ng} / \mathrm{ml}$ in different clinical trials. These dose regimens are still not enough to cure severe and complicated malaria in $100 \%$. It is the antimalarial activity with high killing capability in the first few hours following the first administration of IV AS that is critical in severe malaria. ${ }^{21 ' 22}$

Artemisinin leads to an autoinduction of drug metabolism, which reduces its own bioavailability. ${ }^{23}$ The plasma drug concentrations of daily AS doses were $1 / 3$ rd less on day 3 than day 1 , suggesting autoinduction of hepatic drug metabolism enzymes. Thus, it is important to avoid low dose and to give high initial IV dose for few days for antiviral effect and to avoid autoinduction of metabolism and less antiviral effect due to low drug levels as it occurs in malaria therapy. ${ }^{24,25}$ In clinical trials of artesunate monotherapy used in dosages of $1-8 \mathrm{mg} / \mathrm{kg}$ intravenously or 600-1200 mg per day orally for 5 days were safe. In combination therapies 4-25 $\mathrm{mg} / \mathrm{kg}$ intravenous AS or 200- $800 \mathrm{mg}$ per day orally for 3 days have been used in treatment of malaria. A longer exposure time to a lower peak blood concentration of an artemisinin derivative is more neurotoxic than a shorter duration of exposure with a higher peak blood concentration. Artemisinin derivatives are well tolerated by patients. Mild and reversible hematological and electrocardiographic abnormalities, such as neutropenia and first-degree heart block have been observed infrequently. ${ }^{26}$ Neurotoxic effects have been repeatedly reported in experiments with mice, rats, and dogs with Artemether and Arteether. A longer exposure time to a lower peak blood concentration of an artemisinin derivative is more neurotoxic than a shorter duration of exposure with a higher peak blood concentration. ${ }^{27}$ There were delayed coma recovery times in Gambian children with malaria who were treated with intramuscular artemether versus intravenous quinine. ${ }^{28}$ Dondorp et al. observed patients with malaria, treated with artesunate were compared with patients treated with quinine. The authors did not find significant differences in terms of neurotoxic symptoms (i.e. times to speak, eat, and sit) between treatment groups. Neurological sequel did not occur after treatment with artesunate. ${ }^{29}$

The PK variability of AS following $120 \mathrm{mg}$ of IV AS with high Cmax occurs with first exposure time and the Cmax variability ranges from 735$1890 \mathrm{ng} / \mathrm{ml}$ (AS+DHA). This variability is 25 fold among different clinical trials. Thus ,there is large inter-individual variability in PK/PD and such low drug concentrations presenting in some patients may explain treatment failure; so low dose regimen of AS to be avoided. ${ }^{30}$ AS and DHA readiness to entering the human RBCs due to higher free drug in the plasma related to less protein binding of AS and DHA in comparison to other artemisinin derivatives. ${ }^{31}$ Patients with severe COVID-19 may have many critical and variable conditions and comorbidities i.e. acute kidney injury, hepatopathy, and multiorgan failure etc. with variable severity scores that may determine the drug's PK/PD characteristics and prognosis. The current dose of 120mg IV AS produce variable Cmax from 735 $1890 \mathrm{ng} / \mathrm{ml}$ with greater inter individual variability is still a lower Cmax. Patients with hyperparasitemia require higher Cmax of about $7500-30,000 \mathrm{ng} / \mathrm{ml}$ or 
$4-8 \mathrm{mg} / \mathrm{kg}$ body weight to efficaciously cover all RBCs within first exposure time of 1-2 hrs. The 4$8 \mathrm{mg} / \mathrm{kg}$ loading dose is safe, as the maximum tolerated dose (MTD) of AS is $240 \mathrm{mg} / \mathrm{kg}$ in rats used for 3 days without neurotoxicity. In a current phase I study with IV AS of $4-8 \mathrm{mg} / \mathrm{kg}$ loading dose was extremely well tolerated in humane volunteers and malaria patients and for the consideration of WHO experts and investigators in the further development and treatment of severe faciparum malaria. $^{32}$

The replication Timescale of coronavirus is known by indirect evidences from tissue culture in that virion entry into cell takes about $\sim 10$ minutes (measured for SARS-CoV1),Eclipse period is $\sim 10$ hours (the duration to make intracellular virions corresponds to duration of replication cycle). The burst size is about $\sim 10^{3}$ virions /virion (measured for MHV coronavirus) and the viruses release after 12-36 hours from the infected cell. ${ }^{33}$

Artesunate is superior to all regards in PK/PD profiles with killing power of $99.99 \%\left(10^{4}\right.$ /cycle $)$ of malaria parasites. Artesunate reduced malaria parasitemia by 10,000 fold (vs quinine 100-1000 fold per asexual life cycle). Antimalarial drugs even with $99.99 \%$ killing power need to be given for at least $>3$ life cycle (7 days) to remove all malaria parasites from the body $\left(10^{12}\right)$, for which ACT containing lumefantrine (which cover $>6$ days) is required following 3 days of high dose artesunate therapy to eliminate all parasites from the body . The current dose regimens of IV AS are appears to be insufficient in treatment of severe malaria, and larger loading dose (4-8mg/kg b.w.) may be used for few days ( $\geq 3$ days) to ensure maximum effect. ${ }^{34}$ This may be followed by sequential administration of lumefantrine which have also anti-SARS-CoV2 effects. Lumefantrine has the prolonged half life of up to 6 days led to a cumulative effect after administration of six $80 \mathrm{mg}$ lumefantrine orally twice daily total of $480 \mathrm{mg}$ over 3 days. The EC50 against SARS-CoV-2 was $23.17 \pm 3.22 \mu \mathrm{M}$, both in plasma and in the lungs and its SI was greater than $4.40 \pm 0.61$.The EC50 of Artesunate has an ideal EC50 value of $12.98 \pm 5.30 \mu \mathrm{M}$ and its SI was 5.10 \pm 2.08. $\quad(1 \mu \mathrm{M} \quad$ Artesunate $=384.4 \mu \mathrm{g} / \mathrm{L} \quad \& 1 \mu \mathrm{M}$ $\mathrm{DHA}=284.4 \mu \mathrm{g} / \mathrm{L})$. Artesunate could inhibit SARSCoV-2 replication in a dose-dependent manner.SARS-CoV-2 nucleoprotein (NP) expression inhibited by artesunate, DHA and lumefantrine. Data revealed that artesunate and lumefantrine might function at the post-entry stage of SARS-CoV-2 by interfering with the intracellular events of the SARS CoV-2 infection cycle. ${ }^{35}$ Another potential option is to add an agent after 3 days of AS therapy like Edaravone which have multiple beneficial effects, such as antiinflammatory, antioxidant, anticytokine, immunomodulatory, anti-fibrotic, anti-apoptotic, anti-necrotic, membrane stabilizing, and organ protective properties for the treatment of moderate to severe COVID-19 induced hyperinflammation instead of steroids or other immunosuppressants which are associated with adverse effects such as delayed viral clearance and secondary bacterial infections. ${ }^{36}$

\section{Conclusion}

Suggested doses and timing of high dose initial IV artesunate administration for the treatment of symptomatic COVID-19 infection

Concentration of artesunate required for antiviral effect is $(>10 \mu \mathrm{M})$. From the experience of treatment of severe falciparum malaria, it is conceivable that, for the treatment of COVID-19, it is best to start with high initial IV dose of Artesunate, i.e. 4$8 \mathrm{mg} / \mathrm{kg} / \mathrm{b} . \mathrm{w}$ or $280-560 \mathrm{mg}$ for a $70 \mathrm{~kg}$ person, given IV bolus infusion at 10-12hour interval to achieve $\geq$ $10 \mu \mathrm{M}$ of serum concentration or $300-3000 \mathrm{ng} / \mathrm{ml}$ or even higher for the first exposure. It should be given for a short course therapy of $\geq$ three days will cover $\geq 7$ replication cycle of COVID-19 at the early stages of symptomatic disease associate with robust viral replication for best results and to prevent progression of disease ; at the same time to avoid autoinduction of drug metabolism leading lower AS concentration and neurotoxicity. Artesunate also has anti-inflammatory, immunomodulatory, antioxidant, anticytokine, multiorgan protective effects may be useful even in 
the later stages of hyperinflammation to reduce morbidity and mortality. Thus, artesunate is a perfect potential agent for the urgent treatment of moderate to severe COVID-19 infection, but can be administered at any stage of the disease course.

\section{Acknowledgement}

We are very much thankful to our family members for their kind cooperation and encouragement for this work for the benefits of COVID-19 patients. We have no any conflict of interest.

\section{Reference}

1. Yujun Tang, Jiajia Liu, Dingyi Zhang, Zhanghao Yu, Jinjun Ji, Changping Wen. Cytokine storm in COVID-19: The current Evidence and treatment strategies. Frontiers in Immunology. 2020; 11:1708. doi.10.3389/fimmu.2020.01708.

2. Sarah D Alessandro, Diletta Seaccabarozzi, Lucia Signorini, Fedrica Perogo, Denise P Ilboudo et al. The use of antimalarial drugs against viral infection. Microorganism. 2020;8;85.doi.10.3390/microorganism. 80/0085.www.mdpi.com/journal/microorgan ism.

3. Tuğçenur Uzun, Orcun Toptas. Artesunate: could be an alternative drug to chloroquine in COVID- 19 treatment? Chinese Medicine. (2020) 15:54. https://doi.org/10.1186/s13020-020-00336-8.

4. $\mathrm{H} \mathrm{Xu}, \mathrm{Y}$ He, X Yang, L Liang, Z Zhang, $\mathrm{Y}$ Ye,X Yang,F Lian,L Sun. Antimalarial Agent Artesunate Inhibit TNF- $\alpha$-induced Production of Proinflammatory Cytokines via Inhibition of NF-k $\beta$ and P13Kinase/Akt Signal Pathway in Human Rheumatoid Arthritis Fibroblast-like Synoviocytes. Rheumatology.2007;46:920-926.

5. Wenosch C, Linnau KF,et al.Plasma level of the IL- 6 found persistently with plasmodium falciparum malaria.J Infect Dis. 1999;179: 747-750.

6. L Jiang, et al. Artesunate attenuates lung injury in paraquat intoxicated rats via downregulation of inflammatory cytokines. Cli Lab.2015; 61(11):1601-1607).

7. Chang Cheng, W. Eugene Ho, Fera Y. Goh, Shou Ping Guan, Li Ren Kong, et al. AntiMalarial Drug Artesunate Attenuates Experimental Allergic Asthma via Inhibition of the Phosphoinositide 3-Kinase/Akt Pathway. PLoS ONE. June 2011; 6 (6): e20932. www.plosone.org.

8. Mei Kuang, Yanyan Cen, Rongxin Qin, Shenglan Shang, Zhaoxia Zhai. et al. Artesunate Attenuates Pro-Inflammatory Cytokine Release from Macrophages by Inhibiting TLR4-Mediated Autophagic Activation via the TRAF6-Beclin1-PI3KC3 Pathway. Cell Physiol Biochem 2018; 47:475-488. DOI: 10.1159/000489982.

9. Dorothy HJ Cheong, Danel WS Tan, Fred WS Wong, Thai Tan. Antimalarial drug artemisinin and its derivatives for the treatment of respiratory diseases. Pharmacological Research.2020; 158: 104901.

10. Thomas Efferth, Marta R, Romero, Dano G Wolf, Thomas Stamminger, Jose J, G Mann. The antiviral Activities of Artemisinin and Artesunate .Clinical Infectious Disease.2008; 47; 804-811.

11. Rong Jun Wan, Yue Hong Li. Effects of Artesunate prevent nephritis via the Toll-like receptor 4/nuclear factor- $\kappa \mathrm{B}$ signaling pathway in rats. Molecular Medicine Reports 16: 6389-6395, 2017. Doi: 10.3892/mmr.2017.7362.

12. Lin Y,Wu F,Xie Z,Song X,Zhu X,Zhu Q, Wei J,Tan S,Liang L.Ging B L. Clinical study of artesunate in the treatment of coronavirus disease 2019.Europe PMC. 01 Apr 2020, 32(4):417-420. DOI: 10.3760/cma.j.cn121430-2020031200412. PMID: 3252734.

13. Skinner TS, Manning LS, Johnston WA, Davis TM. In vitro stage specific sensitivity of plasmodium falciparum to quinine and 
artemisinin drugs. Inf J Parsite.1996; 26:519-525.

14. Clinical Monograph. Aretesunate. An advance in malaria management. Scientific communication.1996;ISBN Scientific communication.962-524.(C33-0

15. Alin MH, Bjorkman A. Concentration and time dependence of artemisinin efficacy against plasmodium falciparum in vitro. AM J Trop Med Hyg.1994; 50; 771-776.

16. Li Q, Milhous WK, Weina PJ. Fatal neurotoxicity of artemisinin derivatives is related to drug pharmacokinetics profiles in animal species. Curr Topic Toxicol.2006; $3: 1-16$.

17. White NJ. Clinical pharmacokinetics and pharmacodynamics of artemisinin and its derivatives. Trans R Soc Trop Med Hyg.1994; 88 :( suppl-1) S41-43.

18. Ilett, KF, Batty KT. Powell S. M Binh, T Q, Thu 1., Phuong HL, Hung NC,Davis TM. The pharmacokinetic properties of intramuscular artesunate and rectal Dihydroartemisinine in uncomplicated falciparum malaria. Br. J. Clin. Pharmacol.2002; 53, 23-30.

19. Hien TT, Davis TM, Chuog LV et al. Comparative pharmacokinetics of intramuscular artesunate and artemeether in patients with severe falciparum malaria. Antimicrob Agents Chemother.2004; 48:4234-4239.

20. Suputtamanskhoi Y, Newton PN, Angus B, Tejaisavadharm P. et al. A comparison of oral artesunate and artemether antimalarial bioavailability in acute falciparum malaria.Br J Cli Pharmacol.2001;52:655-661.

21. Tiang JB, Li GQ; Guo, XB Kong YC. Antimalarial activity of mefloquine and qinghaosu. Lancet. Sat. Aug.1982; 2:285288.

22. White NJ. Antimalarial pharmacokinetics and treatment regimens. 1992; 34:1-10.

23. Gordi T, Xie R, Huong NV, Huong DX, Karlsson MO, Ashton M. A semiphysiological pharmacokinetic model for artemisinin in healthy subjects incorporating autoinduction of metabolism and saturable first pass hepatic extraction. $\mathrm{Br}$ J Clin Pharmacol .2005; 59:189-98.

24. Ashtan M,Hai TN, Sy ND, Huang, DX, Van Huang N, Nieu NJ, Cong LD. Artemisinin pharmacokinetics is time dependent during repeated oral administration in healthy male adults. Drug Metab Dispos.1998;26:25-27.

25. Khanh, NX; de Vries , PJ Ha, LD Van, Boxtel CJ, Koopmans R, Kager PA. Decline concentrations of Dihydroartemisinine in plasma during 5-day oral treatment with artesunate for falciparum malaria. Antimicrob Agents Chemother .1999; 43:690-692.

26. Barradell LB, Fitton A. Artesunate: a review of its pharmacology and therapeutic efficacy in treatment of malaria. Drugs 1995; 50:714-41.

27. Li QC, Mog SR, Si YZ, Kyle DE, Gettayacamin M, Milhous WK. Neurotoxicity and efficacy of arteether related to its exposure times and exposure levels in rodents. Am J Trop Med Hyg 2002; 66:516-25.

28. van Hensbroek MB, Onyiorah E, Jaffar S, et al. A trial of artemether or quinine in children with cerebral malaria. N Engl J Med 1996; 335: 69-75.

29. Dondorp A, Nosten F, Stepniewska K, Day N, White N; South East Asian Quinine Artesunate Malaria Trial (SEAQUAMAT) Group. Artesunate versus quinine for treatment of severe falciparum malaria: a randomised trial. Lancet 2005; 366:717-2

30. Karbwang J; Na-Bangchang K, Thanavibut A, Molunto P. Plasma concentrations of artemether and its major plasma metabolite Dihydroartemisinine following a 5 day regimen of oral artemether in patients with uncomplicated falciparum malaria. Ann Trop Med Parsit.1998; 92:31-36 
31. Li Q, Xie LH; Haeberle A, Zhang J, Weina $P$. The evaluation of radiolabeled artesunate in tissue distribution in rats and protein binding in human. Am $\mathrm{J}$ Trop Med Hyg. 1006; 75:817-826.

32. Li Q, Milhous WK, Weina PJ. Artemisinin in malaria therapy. Overseas press (India) Pvt. Ltd. First Edn.2003.p-10, 82, 84,98.

33. YM Bar-On, A Flamholz, R Phillips, R Milo. Science Forum .SARS-CoV-2 (COVID-19) by the numbers. eLife.2020; 9:e57309. DOI https://doi.org/10.7554/eLife.57309.p- 1- 15.

34. Pradhan B. Some considerations in antimalarial chemotherapy of severe falciparum malaria. J Evid Based Med Healthc.2017; 4(13):743 748. doi.10.18410/jebmh/2017/144.

35. Ruiyuan Cao, Hengrui Hu,Yufeng Li, Xi Wang, Mingyue $\mathrm{Xu}$, Jia Liu. et al.AntiSARS-CoV-2 Potential of Artemisinins InVitro. ACS Infect Dis. July 31,2020. https://dx.doi.org/10.1021/acsinfecdis.0c005 22.

36. Dr Butungeshwar Pradhan et al. Edaravone: A Free Radical Scavenger with Multiple Pleotropic Actions can be a Potential Game Changer Agent in Prevention and Alleviation of COVID-19 - Induced Cytokine Storm. JMSCR. July 2020; 8 (7): P- 227- 236. 